\title{
P0517- FGM Improved the Blood Glucose control and QOL of Type 2 diabetes in outpatients
}

Binhong Duan, Dan Wang.

Endocrinology Department of Heilongjiang Provincial Hospital, Harbin, Heilongjiang

Province, China 150036

Backgroud: The Freestyle Libre flash glucose monitoring (FGM) system is similar to conventional continuous glucose monitoring (CGM) systems, but a few significant differences exist. FGM sensors are factory calibrated and therefore do not require calibration with blood glucose testing over their 14-day lifespan. FGM lacks alarm features and connectivity with continuous subcutaneous insulin infu- sion (CSII) devices.The accuracy and usability of FGM have been validated in patients with both type I and type 2 diabetes.

Objective: To explore the effect of FGM on blood glucose control and QOL of Type 2 diabetes in outpatients.

Methods: A total of 60 patients Blood Glucose control and QOL of Type 2 diabetes in outpatients were assigned randomly to the FGM intervention $(n=30)$ or the standard care (SMBG) group $(n=30)$ and followed up for 12 weeks. Both groups were instructed to adjust their insulin doses in face-to-face visits at Iweek, 2 weeks, 4 weeks and 12 weeks. Quality of life (DDS), $\mathrm{HbAlc}$, and frequency of hypoglycemic events were evaluated.

Results: 12 weeks later, compared to the baseline(Table I), SMBG group and FGM group had a significant declination in $\mathrm{HbAlc}$, from $8.5 \pm \mathrm{I} .4 \%$ to $7.1 \pm 1.6 \%$, from $8.4 \pm 1.2 \%$ to $6.9 \pm 1.6 \%, \quad(P<0.05)$, but there was no significant difference in both groups, $(P>0.05)$. The incidence of hyperglycemia was significantly less in FGM group $(P<0.05)($ Table2). Similarly, DDS was significantly improved in FGM group than in SMBG group $(P<0.05)$ (Table 3$)$.

\begin{tabular}{llll}
\multicolumn{2}{l}{ Table 1. Baseline characteristics } & & \\
\hline & FGM & SMBG & P value \\
\hline age & $57.8 \pm 12.3$ & $58.6 \pm 10.8$ & $>0.05 \%$ \\
Sex(M/F) & $14 / 16$ & $15 / 15$ & $>0.05 \%$ \\
Duration(years) & $7.8 \pm 2.4$ & $7.6 \pm 2.5$ & $>0.05 \%$ \\
\hline HbA1c (\%) & $8.4 \pm 1.2$ & $8.5 \pm 1.4$ & $>0.05 \%$
\end{tabular}

Table 2. Changes in HbA1c and hypoglycemic episode over 12 weeks

\begin{tabular}{llll}
\hline & FGM & SMBG & P value \\
\hline HbA1c (\%) & $6.9 \pm 1.6$ & $7.1 \pm 1.6$ & $>0.05 \%$ \\
$\begin{array}{l}\text { Incidence of } \\
\text { Hypoglycemia }\end{array}$ & 3.57 & 1.12 & $<0.05 \%$ \\
\hline
\end{tabular}

Table 3. Difference in DDS over 12 weeks

\begin{tabular}{llll}
\hline & FGM & SMBG & P value \\
\hline Feeling overwhelmed & $1.08 \pm 0.6$ & $3.27 \pm 0.74$ & $<0.05 \%$ \\
Failing routine & $1.57 \pm 0.93$ & $3.23 \pm 1.20$ & $<0.05 \%$ \\
Energy-consuming & $0.91 \pm 0.30$ & $3.35 \pm 1.31$ & $<0.05 \%$ \\
Depressed & $1.47 \pm 1.04$ & $3.24 \pm 1.21$ & $<0.05 \%$ \\
Frequent testing & $1.25 \pm 0.97$ & $3.36 \pm 1.18$ & $<0.05 \%$ \\
\hline
\end{tabular}

Conclusion: FGM was associated with an enormous increase in treatment satisfaction, less hypoglycemia and similar glucose control in outpatients with insulin injection. 